\title{
Morphology, Microstructure, and Hydrogen Content of Carbon Nanostructures Obtained by PECVD at Various Temperatures
}

\author{
M. Acosta Gentoiu, ${ }^{1,2}$ R. Betancourt-Riera, ${ }^{3}$ S. Vizireanu, ${ }^{4}$ I. Burducea, ${ }^{5}$ V. Marascu, ${ }^{2,4}$ \\ S. D. Stoica, ${ }^{4}$ B. I. Bita, ${ }^{2,6}$ G. Dinescu, ${ }^{4}$ and R. Riera ${ }^{1}$ \\ ${ }^{1}$ Departamento de Investigación en Física, Universidad de Sonora, Apdo. Postal 5-088, 83190 Hermosillo, SON, Mexico \\ ${ }^{2}$ Faculty of Physics, University of Bucharest, 405 Atomistilor Street, 077125 Măgurele, Romania \\ ${ }^{3}$ Instituto Tecnológico de Hermosillo, Avenida Tecnológico S/N, Col. Sahuaro, 83170 Hermosillo, SON, Mexico \\ ${ }^{4}$ National Institute for Lasers, Plasma and Radiation Physics, Atomistilor No. 409, P.O. Box MG-36, Măgurele, Bucharest, Romania \\ ${ }^{5}$ Horia Hulubei National Institute of Physics and Nuclear Engineering, 30 Reactorului Street, Măgurele, 077125 Ilfov, Romania \\ ${ }^{6}$ National Institute for Red in Microtechnologies, 126 A Erou Iancu Nicolae Street, Voluntari, Romania
}

Correspondence should be addressed to S. Vizireanu; s_vizi@infim.ro

Received 16 March 2017; Revised 6 July 2017; Accepted 27 July 2017; Published 11 September 2017

Academic Editor: Yoke K. Yap

Copyright (C) 2017 M. Acosta Gentoiu et al. This is an open access article distributed under the Creative Commons Attribution License, which permits unrestricted use, distribution, and reproduction in any medium, provided the original work is properly cited.

\begin{abstract}
Carbon nanostructures were obtained by acetylene injection into an argon plasma jet in the presence of hydrogen. The samples were synthesized in similar conditions, except that the substrate deposition temperatures $T_{D}$ were varied, ranging from 473 to $973 \mathrm{~K}$. A strong dependence of morphology, structure, and graphitization upon $T_{D}$ was found. We obtained vertical aligned carbon nanotubes (VA-CNTs) at low temperatures as $473 \mathrm{~K}$, amorphous carbon nanoparticles (CNPs) at temperatures from about 573 to $673 \mathrm{~K}$, and carbon nanowalls (CNWs) at high temperatures from 773 to $973 \mathrm{~K}$. Fourier transform infrared spectroscopy, scanning electron microscopy, transmission electron microscopy, elastic recoil detection analysis, X-ray photoelectron spectroscopy, and Raman spectroscopy were used to substantiate the differences in these material types. It is known that hydrogen concentration modifies strongly the properties of the materials. Different concentrations of hydrogen-bonded carbon could be identified in amorphous CNP, VA-CNT, and CNW. Also, the $\mathrm{H}$ : C ratios along depth were determined for the obtained materials.
\end{abstract}

\section{Introduction}

Carbon nanostructures have attracted special attention as very promising materials for nanotechnology. The three kinds of carbon hybrid bonds, $\mathrm{sp}^{1}, \mathrm{sp}^{2}$, and $\mathrm{sp}^{3}$, different C-C, C-O, and $\mathrm{C}-\mathrm{H}$ bonding arrangement, and dimensional network make the carbon-containing films a complex structure with a wide variety of allotropy and applications [1]. Formations like quasi-zero-dimensional (Q0D) spheres/nanoparticles [2, 3], quasi-one-dimensional (Q1D) wires/rods/tubes $[4,5]$, and quasi-two-dimensional (Q2D) walls/flakes/sheets nanocarbon were reported [6].

The Q2D system has been in the focus of investigations in the past years. It opened a new window to quantum Hall effect, solar cells, biosensors, and electrodes for energy storage devices [7]. Many of the recent studies were devoted to vertically oriented nanosheets or carbon nanowall (CNW) materials, described as graphene sheets forming a self-supported network of vertical wall structures, randomly oriented, with thicknesses of a few tens of nanometers [8]. Because of their graphene-like structure with high surfacearea-to-volume ratio, CNWs have many potential applications such as electrochemistry, catalyst supports, and electrochemical capacitors [9]. In addition, other useful structures are vertically aligned carbon nanotubes (VA-CNTs) $[5,10]$, in forms of single-walled nanotubes (SWNTs) or multiwalled nanotubes (MWNTs) [5], and amorphous carbon nanoparticles (CNPs) in different hydrogen-carbon concentrations $[11,12]$.

A PECVD system [13-15], based on low pressure expanding RF plasma jet generated in argon, in the presence of acetylene and hydrogen mixture, was used for the growth 
of tree type nanostructures (VA-CNT, CNP, and CNW). Radicals are formed by dissociation of acetylene through collisions into the plasma jet and are further transported to the substrate, where they sustain the growth process. We studied the influence of deposition temperature $\left(T_{D}\right)$ at the substrate, ranging from 473 to $973 \mathrm{~K}$, on the properties of the synthesized carbon nanomaterials. The resulting samples were characterized by several techniques: scanning electron microscopy (SEM) for the material morphology and Fourier transform infrared spectroscopy (FTIR), transmission electron microscopy (TEM), X-ray photoelectron spectroscopy (XPS), and Raman spectroscopy for a better understanding of the structure and chemical composition. Particularly, the hydrogen concentration can be related to the physical and chemical properties, and its amount could modify the $\mathrm{C}-\mathrm{C}$ network along the depth of the material. However, the hydrogen concentration is difficult to identify by usual techniques. Herein, elastic recoil detection analysis (ERDA) reveals the hydrogen concentration and its in-depth profile of nanomaterial when different synthesis temperatures were used.

\section{Experimental}

2.1. Synthesis of Carbon Nanomaterials. The carbon nanostructures (CNW, VA-CNT, and CNP) that we discuss in the paper were obtained by plasma enhanced chemical vapor deposition (PECVD) in the same setup through reactive hydrocarbon/argon/hydrogen plasma jet [13-15]. In the synthesis of carbon materials, the following parameters were fixed: gas flow ratios $\mathrm{Ar}: \mathrm{H}_{2}: \mathrm{C}_{2} \mathrm{H}_{2}=1050: 25: 1 \mathrm{sccm}$, pressure of $120 \mathrm{~Pa}$, RF power of $300 \mathrm{~W}$, and deposition time of $60 \mathrm{~min}$. The substrates consisted of Si (double polished transparent to IR) or Si wafers covered by $\mathrm{SiO}_{2}$, in the absence of any catalyst. They were collocated downstream at $5 \mathrm{~cm}$ distance with respect to the injection ring. The deposition temperature measured at the substrate was varied at intervals of $100 \mathrm{~K}$ in the range $473-973 \mathrm{~K}$.

2.2. Techniques of Characterization. The chemical change that occurs in the nanomaterials was analyzed with Jasco FTIR-6300 Type A spectrometer, in transmission mode, in the range of $600-1900 \mathrm{~cm}^{-1}$. For the FTIR investigations, the CNW samples were grown on IR highly transparent double polished thin silicon substrates. The measurements were performed in transmission mode, with $4 \mathrm{~cm}^{-1}$ resolution, using 1024 accumulations to improve the transmitted signal strength of the materials. A fast Fourier filter was applied, resulting in an acceptable signal-to-noise ratio in the spectra. A LabRam HR apparatus was used to obtain the Raman spectrum with $632.8 \mathrm{~nm}$ wavelength. The $\mathrm{CNW}$ samples were characterized by TEM using a JEOL JEM 2010 microscope, operating at $200 \mathrm{kV}$ acceleration voltage. We also used a high resolution FEG-SEM instrument (FEI Nova Nano SEM 630) at $10^{-4}$ mbar vacuum.

The ERDA measurements were performed using $3 \mathrm{MeV}$ ${ }^{4} \mathrm{He}^{2+}$ ions from a $3 \mathrm{MV}$ Tandetron ${ }^{\mathrm{TM}}$ accelerator [16]. The used current density was about $5 \mathrm{nA} / \mathrm{mm}^{2}$, the beam spot was $1 \mathrm{~mm}^{2}$, and the chamber vacuum was $10^{-6} \mathrm{mbar}$. The ion current was kept low to avoid sample heating. The target holder was positive biased at $300 \mathrm{~V}$ in order to prevent secondary electrons emission. The energy of hydrogen recoil was recorded using an AMETEK type BU-012-050-100 solidstate detector with a solid angular acceptance of $1 \mathrm{msr}$ that was placed at a recoiling angle of $30^{\circ}$ to the beam normal. The incident beam angle and the exit angle as measured from the normal to the sample surface were both $75^{\circ} .12 \mu \mathrm{m}$ thick Mylar absorber foil was located in front of the ERDA detector to separate hydrogen recoils hitting the ERDA detector from scattered He ions. The ERDA spectra were simulated using SIMNRA software package [17]. The density of the area or thin film units is the natural units for ion beam analysis, since the energy loss is measured in $\mathrm{eV} /\left(\right.$ atoms $\left./ \mathrm{cm}^{2}\right)$, and one monolayer is of the order of $10^{15}$ atoms $/ \mathrm{cm}^{2}$ [18]. The depth scale is given in thin film units of $10^{15}$ atoms $/ \mathrm{cm}^{2}$, while the depth of measurement is around $900 \mathrm{~nm}$ in the nanocarbon material.

\section{Results and Discussions}

3.1. SEM Investigation. The morphologies of carbon nanomaterials deposited at different $T_{D}$ ranging from 473 to $973 \mathrm{~K}$ are shown through SEM images in Figures 1(a)-1(f). The morphology considerably changes with $T_{D}$ : VA-CNT at $473 \mathrm{~K}$ (Figure 1(a)), CNP at both 573 and $673 \mathrm{~K}$ (Figures 1(b) and 1(c), resp.), and CNW from 773 to $973 \mathrm{~K}$ (Figures 1(d)-1(f)).

We can see that, at $473 \mathrm{~K}$ VA-CNT, there is a mean diameter of about $47 \mathrm{~nm}$ and length of $4.2 \mu \mathrm{m}$. The increase of $T_{D}$ from 573 to $673 \mathrm{~K}$ produces a CNP granular material with a growing diameter of particles from around 58 to $147 \mathrm{~nm}$, with films thicknesses of $1.7 \mu \mathrm{m}$ and $2.1 \mu \mathrm{m}$, respectively. At $773 \mathrm{~K}$, we can see an incipient growth of flake-like nanostructures (edges' length of about $0.2 \mu \mathrm{m}$ ). The cross-sectional investigation (not shown here) indicates vertical orientation of the flakes distributed in a layer of around $3.2 \mu \mathrm{m}$ thickness. At $873 \mathrm{~K}$, the flakes are already well individualized with lengths of about $1 \mu \mathrm{m}$ between their interconnections and $3.4 \mu \mathrm{m}$ in height. Also, we can observe secondary carbon nanowalls branched to the main walls. The morphology is preserved at higher temperature of $973 \mathrm{~K}$, but the layer thickness increases to $4.5 \mu \mathrm{m}$.

3.2. TEM Characterization of CNW. TEM images, shown in Figures 2(a) and 2(b), reveal the Q2D graphitic structure of the CNW synthesized at 873 and $973 \mathrm{~K}$, similar to other reports [19]. The edges thickness varies from $8-10 \mathrm{~nm}$ at $873 \mathrm{~K}$ to $3-5 \mathrm{~nm}$ at $973 \mathrm{~K}$. The interlayer spacing was determined by digital Fourier image analysis, and we obtained $3.7 \pm 0.1 \AA$ and $3.5 \pm 0.1 \AA$. They can be compared to the graphite interlayer $(\sim 3.4 \AA)$. In addition, the graphene sheets are better aligned at higher temperature, as we can observe in Figure 2(c).

The results indicate that, by synthesis at higher $T_{D}$, the edges are narrow with a lower number of graphenes in the walls. In addition, the interlayer spacing decreases and the walls' height increases. This is because, at elevated $T_{D}$, the carbon species that arrive close on the planar region of the walls would reevaporate more easily due to the weak interaction forces compared to the edges' top [20]. Therefore, the growth 


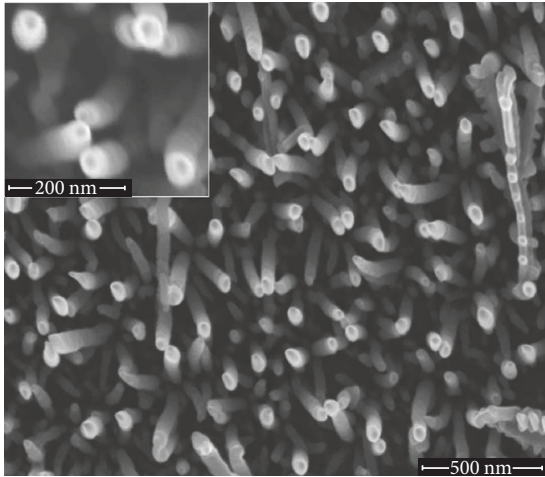

(a)

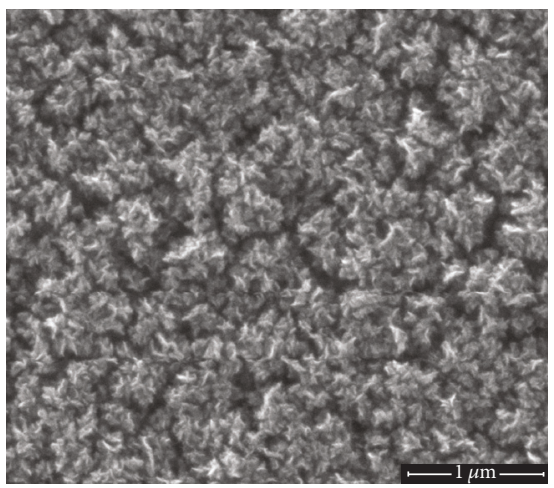

(d)

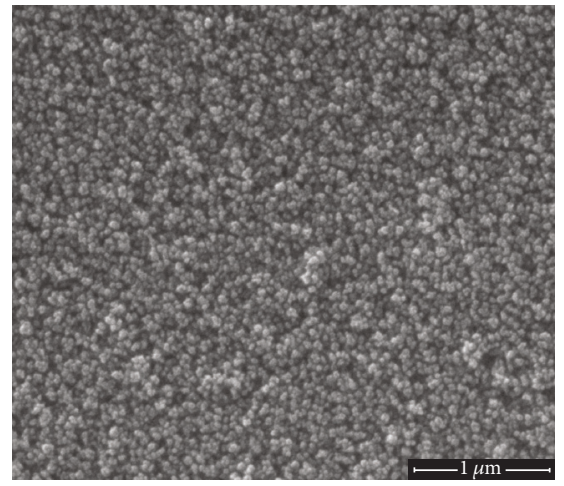

(b)

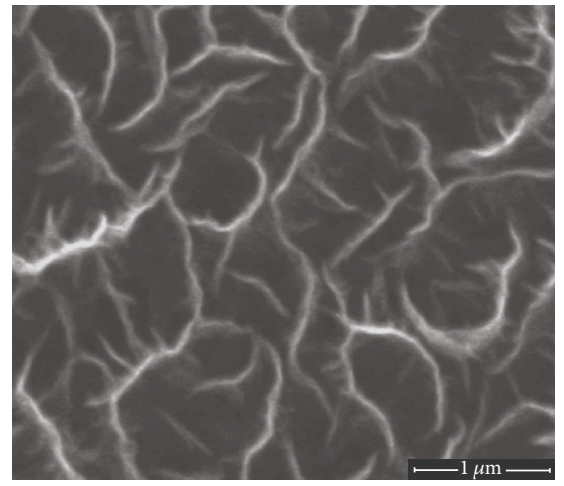

(e)

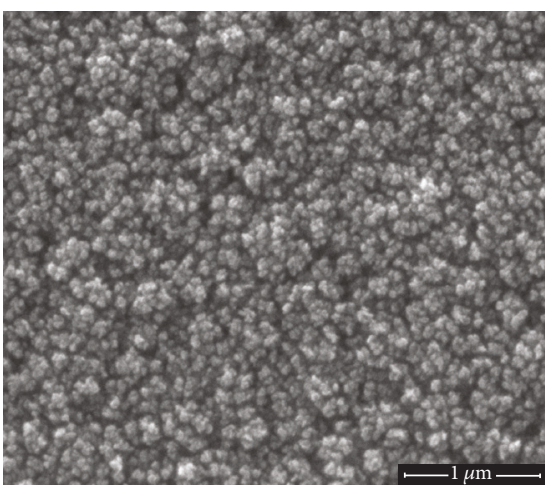

(c)

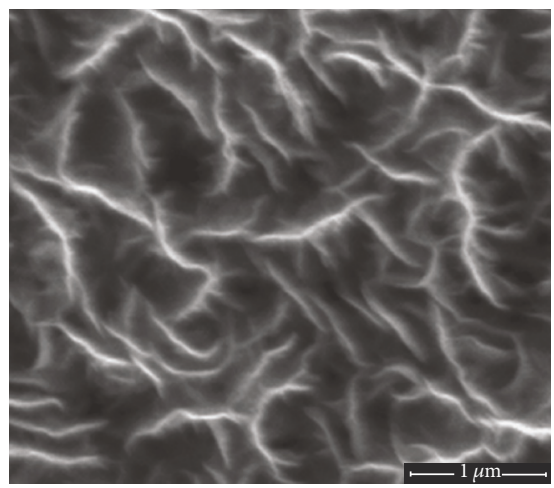

(f)

FIGURE 1: Top view SEM of carbon nanostructures grown on Si substrate using PECVD at different $T_{D}$ : (a) $473 \mathrm{~K}$, (b) $573 \mathrm{~K}$, (c) $673 \mathrm{~K}$, (d) $773 \mathrm{~K}$, (e) $873 \mathrm{~K}$, and (f) $973 \mathrm{~K}$.

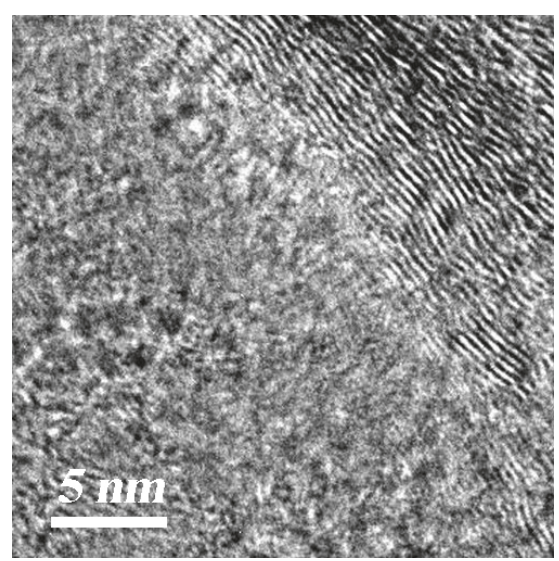

(a)

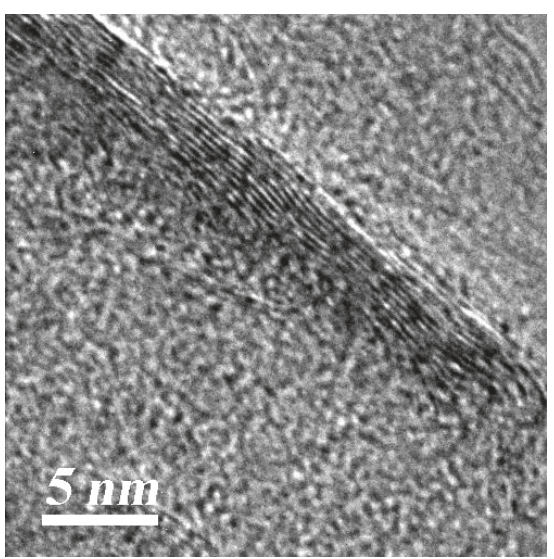

(b)

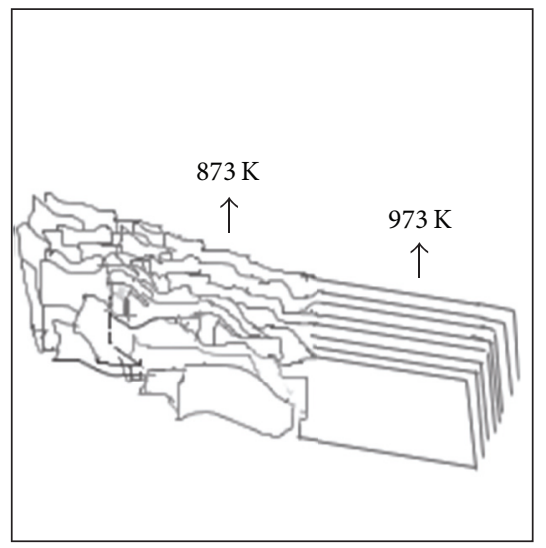

(c)

Figure 2: TEM image of CNW at (a) $873 \mathrm{~K}$ and (b) $973 \mathrm{~K}$ and (c) model of changes from layers stacking with $T_{D}$.

is produced preferentially on the wall top, which presents strong C-C covalent bond. Apparently, at higher temperature, an increase of Van der Waals forces presented between layers occurs, leading to a more aligned and continuous stacking (see Figures 2(a)-2(c)).

3.3. ERDA Measurements. One of the most accurate methods for investigating the hydrogen concentration in materials is ERDA. To our knowledge, comparative ERDA study of different carbon nanomaterials deposited at different $T_{D}$ or the content of hydrogen in $\mathrm{CNW}$ has not been reported before.

In Figure 3, we present the hydrogen concentration dependence upon depth, where the depth is indicated by the number of atomic monolayers (along about $900 \mathrm{~nm}$ of an equivalent homogenous material) sampled by the 


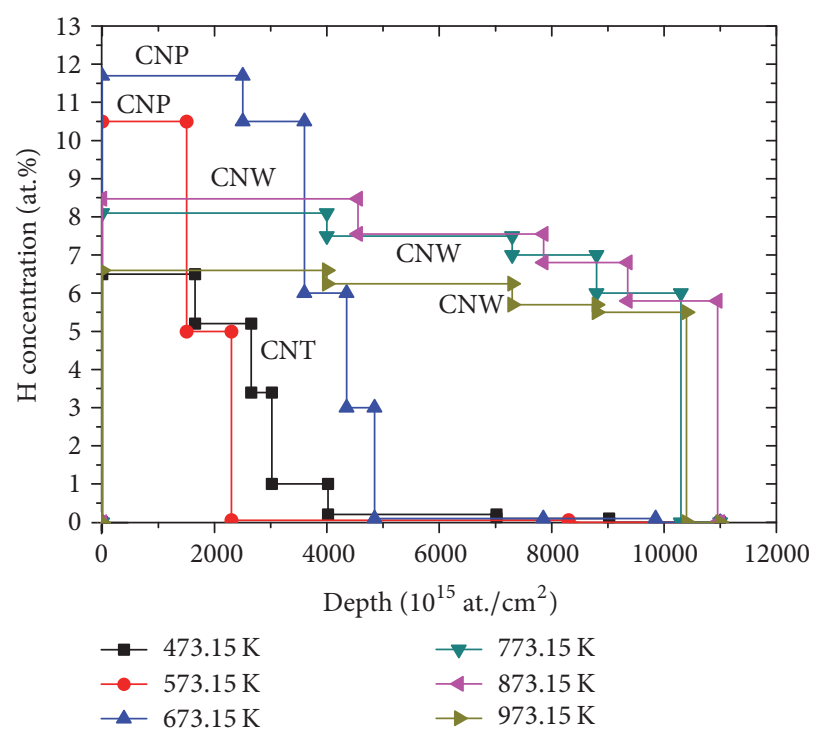

FIGURE 3: In-depth distribution of hydrogen amount by ERDA measurements of the CNT, CNP, and CNW produced at different $T_{D}$.

incident particle beam and the recoiling atoms. Interpretation of these hydrogen profiles is difficult, because the tested materials are not homogeneous. Still, important information can be extracted. One can observe two classes of hydrogen distribution, each class characterized by a different shape. In one kind, the CNT and CNP profiles present a steep decrease at quite small penetration depths. In comparison, in the other class, the CNW material presents a steep decrease of the hydrogen signal after a long plateau. Datta et al. [11] suggest that in amorphous carbon the decrease could be the consequence of the formation of graphitic structure with less $\mathrm{sp}^{3}$ carbon towards the Si substrate. In VA-CNT structure, Gouzman et al. [21] suggested the appearance of amorphous carbon impurities (with high hydrogen content) around the tube. For CNW, there are no reports: we hypothesize that lower energy losses per unit path are valid in the very porous material for the incident ${ }^{4} \mathrm{He}^{2+}$ ions particles, which would travel obliquely across the spatially separated thin graphene stacks. Also, some scattering of the recoiling $\mathrm{H}$ atoms found between sheets and layers can be considered. Anyway, the quite homogeneous concentrations along depth suggest that the hydrogen is attached uniformly to the surface of the vertical wall. Also, the hydrogen content may be associated with (i) dangling bonds on the top of the walls and secondary CNW formed along the plane of the wall, (ii) atomic defects, and (iii) amorphous carbon impurities.

While the real assignment of hydrogen concentration to depth is difficult, the content of hydrogen can be calculated. We exemplified the first report of Naab et al. [22] of hydrogen content on commercially available single-, double-, and multiwalled nanotubes and carbon nanofibers and the report of Gohier et al. [23] which found values between 4 and 8 at.\% of hydrogen in multiwall carbon nanotubes by using high energy of $35 \mathrm{MeV} \mathrm{Cl}^{7+}$ ion beam.

The hydrogen content in CNW was reported before by Jain et al. [24] in percentage of 3\%-5\% by SIMS and Nuclear
TABLE 1: Hydrogen concentration at different deposition temperatures.

\begin{tabular}{lc}
\hline Temperature $(\mathrm{K})$ & H concentration (at.\%) \\
\hline 473 & 4.5 \\
573 & 8.8 \\
673 & 9.7 \\
773 & 7.4 \\
873 & 7.7 \\
973 & 6.2 \\
\hline
\end{tabular}

Reaction Analysis (NRA) methods and Burducea et al. [25] that observed a value of 5.4 at.\% of $\mathrm{H}$ in $\mathrm{CNW}$ measured by NRA using $16.6 \mathrm{MeV}^{19} \mathrm{~F}^{4+}$.

The present results summarized in Table 1 show values ranging from 4.5 to $10 \%$, values obtained by integrating upon depth (areal density) the spectra shown in Figure 3. They reveal that hydrogen is found in the highest amount in CNPs at $673 \mathrm{~K}$ with 9.7 at.\%. By contrast, the hydrogen content in CNT formed at $473 \mathrm{~K}$ is half and in CNW formed at $973 \mathrm{~K}$ it is two-thirds the highest value. By correlating with the morphological and structural results, one concludes that the material with less graphitic formation, as different size agglomerated CNPs, contains more hydrogen compared to the materials with well-defined microstructure, as CNTs and CNWs. We suppose that the defects (dangling bonds) are terminated with hydrogen atoms. However, well-ordered CNT and CNW contain a lower defect density [26, 27] in comparison to amorphous carbon nanoparticles.

3.4. FTIR Analysis in Different Carbon Nanostructures. The dependence of FTIR absorption spectra of the carbon nanomaterials upon temperature for the spectral range $600-1900 \mathrm{~cm}^{-1}$ is shown in Figure 4. Around $800 \mathrm{~cm}^{-1}$, we can see aromatic $=\mathrm{C}-\mathrm{H}$ peak. Those between 700 and 


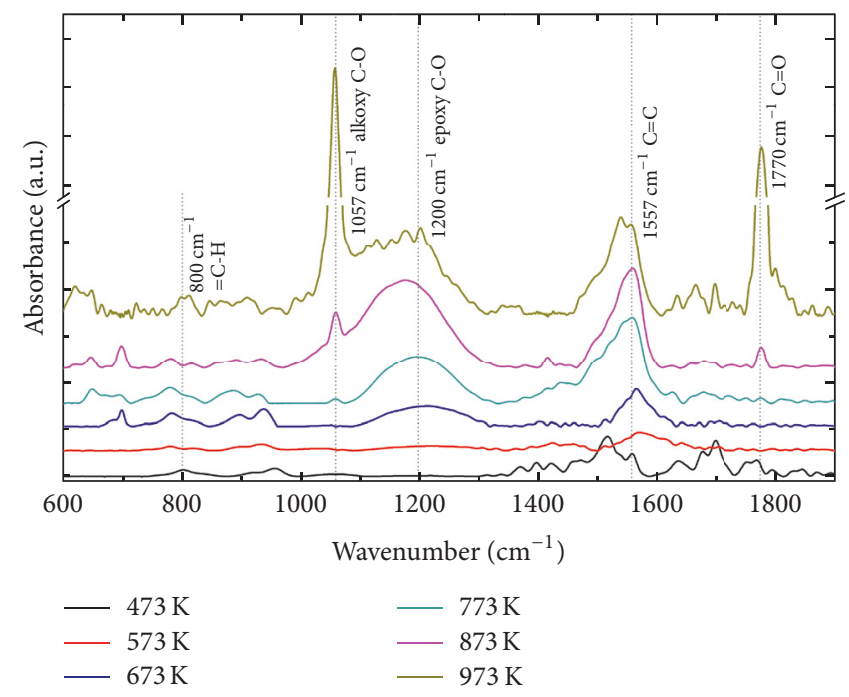

FIGURE 4: Detailed FTIR spectra of carbon nanomaterials obtained at different $T_{D}$ in the $600-1900 \mathrm{~cm}^{-1}$ range.

$960 \mathrm{~cm}^{-1}$ are related to C-H skeleton vibrations with the characteristic appearance of $\mathrm{C}$-H out-of-plane bending structure. Also, a strong peak of aromatic $\mathrm{sp}^{2}$-hybridized $\mathrm{C}=\mathrm{C}$ in plane stretching around $1577 \mathrm{~cm}^{-1}[28]$ is observed.

The bands' assignment to oxygen-containing functional groups is described in the following. The band around 1057, with a prominent peak at $973 \mathrm{~K}$, is usually attributed to alkoxy C-O group [29]. The next band, around $1200 \mathrm{~cm}^{-1}$, could be assigned to epoxy C-O [29]. The peak at $1620 \mathrm{~cm}^{-1}$ belongs either to oxygen compounds or to ring vibrations by the carbon skeleton. The carboxyl or carbonyl $\mathrm{C}=\mathrm{O}$ groups appear in the range $1650-1800 \mathrm{~cm}^{-1}$ [28] with a strong band around $1770 \mathrm{~cm}^{-1}$ at higher $T_{D}$. We can observe the absence or reduction of these oxygen-containing functional groups in CNT and CNP. Therefore, we supposed to find active sites for oxygen bonds essentially at the edges of the graphene layers in the CNW. Similar FTIR studies for carbon nanomaterials were reported before by Vizireanu et al. [14] and Her and Lai in pristine CNT [30]. A peculiarity of the spectra corresponding to high temperature depositions is the huge intensity of the peaks at about 1060 and $1770 \mathrm{~cm}^{-1}$. This suggests a large number of defects like small particle sizes with a high degree of graphitization, which were previously reported by Kurita et al. [31]. These small crystallites can present more edges with unsaturated valences that allow the formation of oxygen groups [32].

\subsection{Comparative Analysis of the $\mathrm{CNW}$ \\ Deposited at 873 and $973 \mathrm{~K}$}

3.5.1. XPS Characterization. To identify elements and bonding type of the CNW materials deposited at 873 and $973 \mathrm{~K}$, $\mathrm{X}$-ray photoelectron spectroscopy analysis was carried out. Figure 5(a) shows the survey spectra with the identified elements, carbon, oxygen, and nitrogen, and their relative atomic concentrations in the inset. The oxygen and nitrogen incorporation could occur during synthesis [33] at moderate vacuum and also during the contact with the environment. We note that the relative concentration of carbon increased from 89.0 to $93.6 \%$, as temperature increased from 873 to $973 \mathrm{~K}$, at the expense of the decrease of the atomic oxygen from 9.3 to $5.4 \%$ and of the nitrogen from 1.7 to $1.0 \%$.

With respect to the bonding types, we studied them for the $\mathrm{CNW}$ at $873 \mathrm{~K}$. We used for high resolution spectra fitting a Voigt function (80\% Gaussian and 20\% Lorentzian) and we established the percent of carbon and oxygen bonds, as can be seen in Figures 5(b) and 5(c), respectively. The C1s region presents a peak at $284.6 \mathrm{eV}$ corresponding to $\mathrm{C}=\mathrm{C} \mathrm{sp}^{2}$ graphitic structure ( $\mathrm{C} 1$ in Figure 5$)$. The other bands were assigned to $\mathrm{sp}^{3} \mathrm{C}$ hybridized to $\mathrm{C}$, single bonded carbon as $\mathrm{C}-\mathrm{OH}, \mathrm{O}-\mathrm{C}-\mathrm{O}$ or $\mathrm{C}-\mathrm{N}$, double bonded carbon as $\mathrm{C}=\mathrm{O}$, $\mathrm{COOH}$, and $\pi-\pi^{*}$ at binding energies of $285.5,286.5,288.4$, 290.0, and $291.5 \mathrm{eV}$, respectively. Figure 5(c) shows the peaks attributed to physical absorbed oxygen, $\mathrm{C}=\mathrm{O}, \mathrm{C}-\mathrm{OH}$ or $\mathrm{C}-\mathrm{O}$, and $\mathrm{COOH}$ at $530.5,531.7,532.8$, and $533.6 \mathrm{eV}$, respectively $[34,35]$. Thus, the results confirm that the oxygen bonding appeared in the FTIR spectra, but with the additional information concerning the adsorbed oxygen.

It is worth noting that, at $873 \mathrm{~K}$, a large part of oxygen is adsorbed $(37.8 \%)$, most probably trapped between the graphene layers, thus affecting the intensity of the Van der Waals forces [36]. In contrast, less oxygen would be adsorbed in the $\mathrm{CNW}$ at $973 \mathrm{~K}$ because of the reduced space between layers, as presented in the TEM images from Figure 2.

3.5.2. Raman Characterization. Raman spectrum for VACNT, CNP, and CNW at 473, 673, and both 873 and $973 \mathrm{~K}$, respectively, is shown in Figure 6. The principal G band appears around $1583 \mathrm{~cm}^{-1}$ indicating $\mathrm{sp}^{2}$ vibrations of hexagonal carbon lattice [37]. This band comes from Stokes Raman scattering with the emission of phonon $E_{2 g}$.

The presence of disorder state, induced by disarrangement of the graphitic planes, creates the sharp $\mathrm{D}^{\prime}$ and $\mathrm{D}$ bands [19]. The $\mathrm{D}^{\prime}$ band (around $1604 \mathrm{~cm}^{-1}$ ), observed only in 


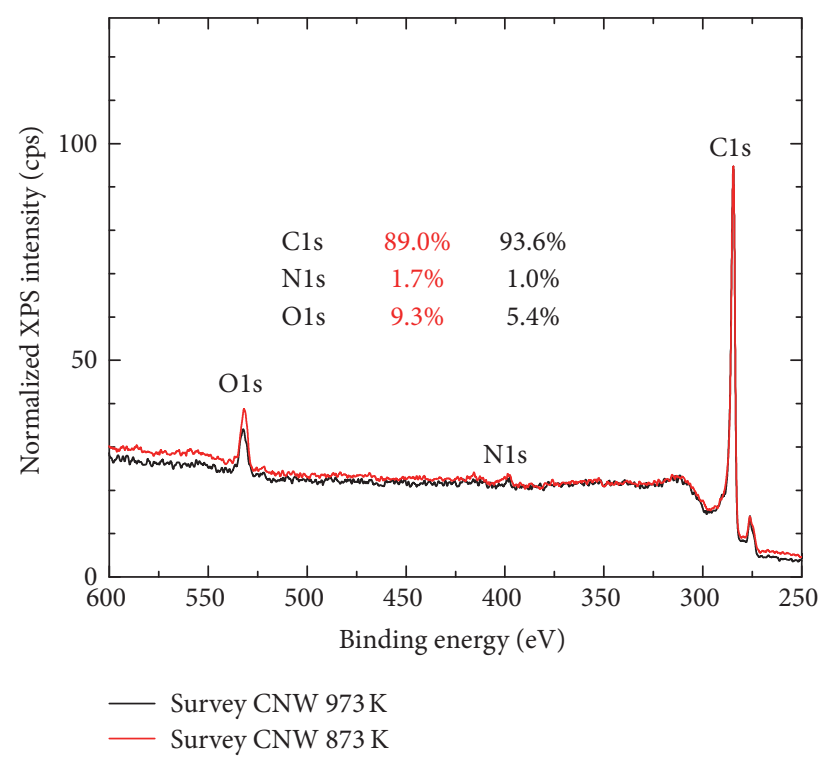

(a)

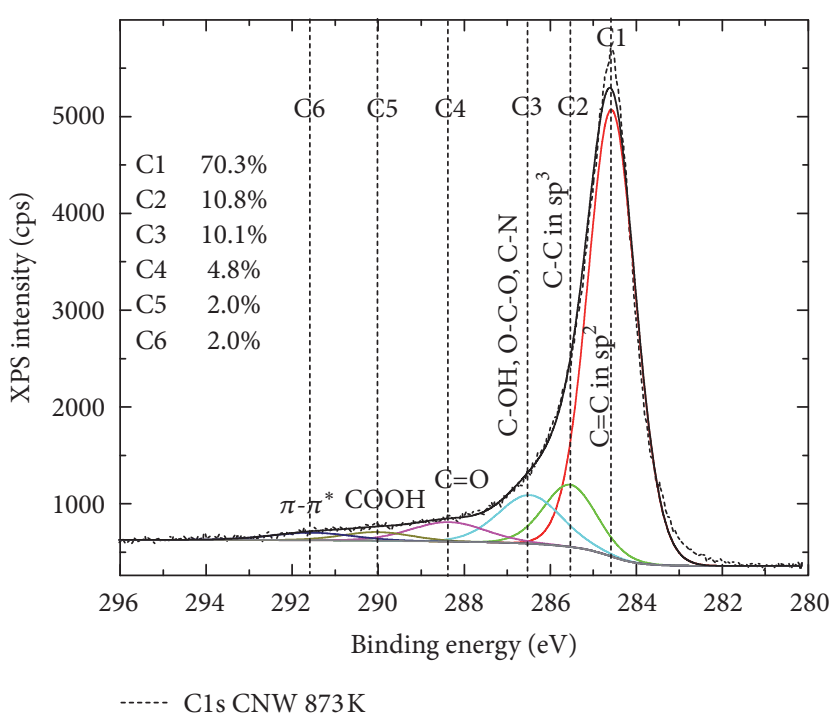

(b)

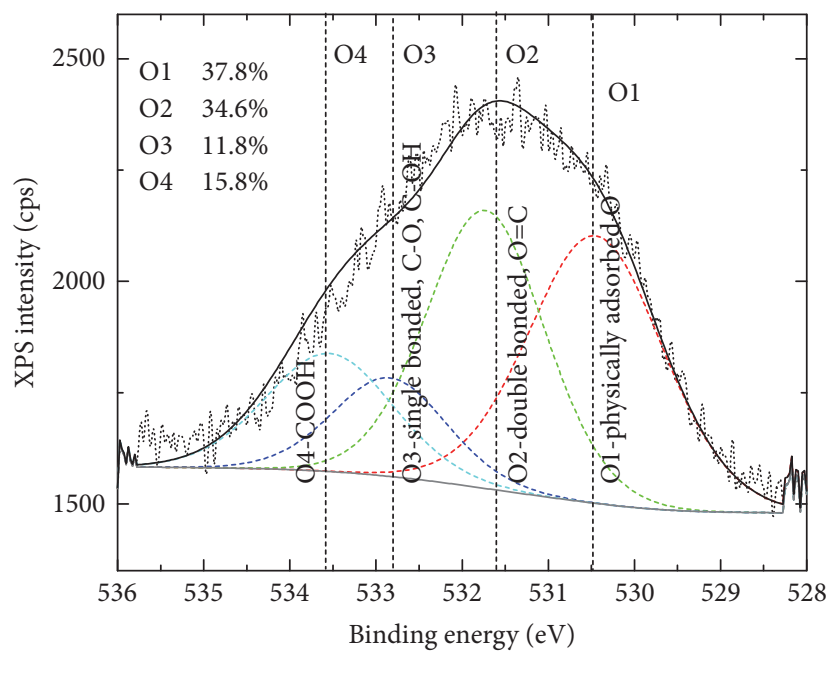

….... O1s CNW 873K

(c)

FIgURE 5: (a) The XPS spectra with C1s, O1s, and N1s bands for the CNW grown at $873 \mathrm{~K}$ (red line) and $973 \mathrm{~K}$ (black line). The figure includes the relative atomic concentrations (percent). In (b) and (c), the deconvolution of C1s and O1s bands is presented, for the CNW at $873 \mathrm{~K}$.

CNW, is related to finite-size graphite crystals and graphene edges. In the region of the higher-order Raman spectrum, we found around $2693 \mathrm{~cm}^{-1}$ the $2 \mathrm{D}$ peak, which is imperceptible at low temperature, in VA-CNT and CNP. For CNW samples, $2 \mathrm{D}$ band is related to the number of graphene layers [38], defect density [20], and the stacking order and orientation of the graphene sheets [39].

The D band (around $1327 \mathrm{~cm}^{-1}$ ) in the CNW describes the disorder state created by finite crystallite size and different orientations of the graphitic domains, defects, grain boundaries, and edges [37]. It is related to the double resonance Raman scattering process. The ratio of intensities of the $\mathrm{D}$ and $\mathrm{G}$ bands, $I(\mathrm{D}) / I(\mathrm{G})$, is often used to evaluate the degree of disorder in graphitic formation and takes a minimum value for $\mathrm{CNT}$ and amorphous $\mathrm{CNP}$ (with value 1 ). In the case of CNW, $I(\mathrm{D}) / I(\mathrm{G})$ ratio increases from about 1.5 to 2.1 by changing $T_{D}$ from 873 to $973 \mathrm{~K}$. By increasing $T_{D}$, we observed an increase of $\mathrm{CNW}$ dimensions in the SEM investigations, accompanied with increasing $I(\mathrm{D}) / I(\mathrm{G})$ ratio in agreement with Kurita et al. [31] who observed a linear dependence between CNW size (length, thickness, and crystallite size) and Raman intensity ratio.

\section{Conclusions}

Carbon nanomaterials were obtained by PECVD technique using RF argon plasma jet injected with acetylene precursor 


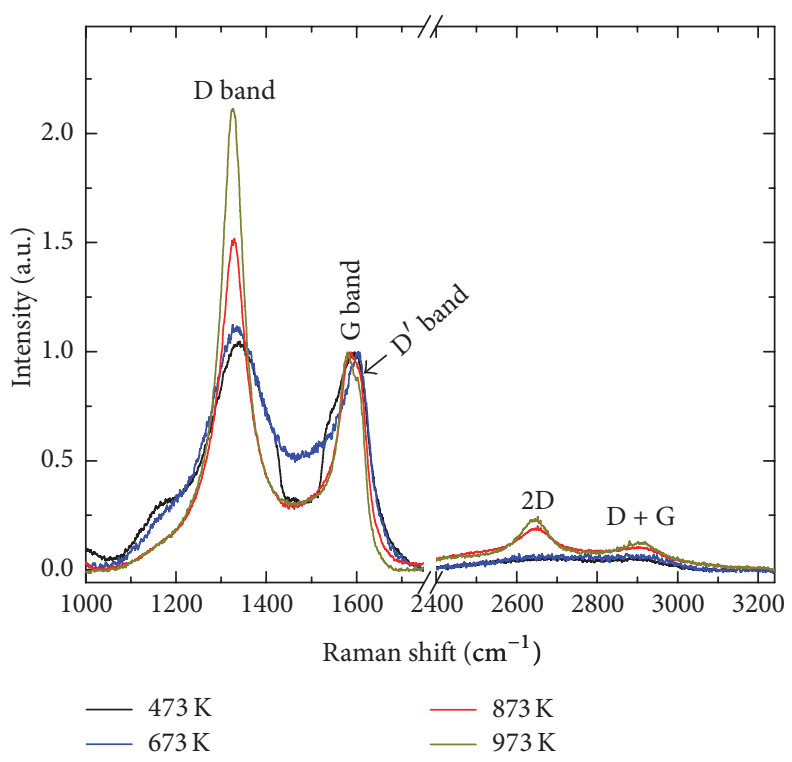

Figure 6: Raman spectra from VA-CNT, CNP, and CNW deposited at 473, 673, 873, and $973 \mathrm{~K}$.

and hydrogen. The morphology, microstructure, and chemical composition of the materials are dependent upon $T_{D}$ employed during the deposition. At low temperature, $473 \mathrm{~K}$, the formation of VA-CNT due to the plasma activation was noted. In the range of 573-673 K, only amorphous CNPs were obtained. Higher temperatures produced a structural modification from amorphous carbon film towards graphitic arrangement, enhancing the formation of CNW material above $773 \mathrm{~K}$. The CNW, unlike the other materials, presented a homogenous hydrogen concentration along the depth. Also, it presented a reduction of the interlayer spacing from 873 to $973 \mathrm{~K}$ with the respective decrease of absorbed oxygen between the layers. Therefore, carbon nanomaterials with desirable quality of thickness, $\mathrm{H}: \mathrm{C}$ ratio, and graphitization can be grown by varying $T_{D}$ by the PECVD method.

\section{Conflicts of Interest}

The authors declare that they have no conflicts of interest.

\section{Acknowledgments}

This work was supported by the Center for Nanoscience and Nanotechnology, UNAM, Mexico, and by the Romanian National Authority for Research and Innovation CNCSUEFISCDI in the frame of Nucleus Programme 4N/2016 and Project no. PN-II-RU-TE-2014-4-2035. The authors would like to thank Dr. C. E. Grigorescu, Dr. R. Mora, Dr. F. Ruiz, R. Garcia, and G. A. Hirata for their help with some of the measurements.

\section{References}

[1] Y. Wu, B. Yang, B. Zong, H. Sun, Z. Shen, and Y. Feng, "Carbon nanowalls and related materials," Journal of Materials Chemistry, vol. 14, no. 4, pp. 469-477, 2004.
[2] J. Yu, E. G. Wang, and X. D. Bai, "Electron field emission from carbon nanoparticles prepared by microwave-plasma chemicalvapor deposition," Applied Physics Letters, vol. 78, no. 15, pp. 2226-2228, 2001.

[3] N. Rao, S. Girshick, J. Heberlein et al., "Nanoparticle formation using a plasma expansion process," Plasma Chemistry and Plasma Processing, vol. 15, no. 4, pp. 581-606, 1995.

[4] K. H. Chen, C. T. Wu, J. S. Hwang et al., "Electron beam induced formation of carbon nanorods," Journal of Physics and Chemistry of Solids, vol. 62, no. 9-10, pp. 1561-1565, 2001.

[5] M. Chhowalla, K. B. K. Teo, C. Ducati et al., "Growth process conditions of vertically aligned carbon nanotubes using plasma enhanced chemical vapor deposition," Journal of Applied Physics, vol. 90, no. 10, pp. 5308-5317, 2001.

[6] J. J. Wang, M. Y. Zhu, R. A. Outlaw et al., "Free-standing subnanometer qraphite sheets," Applied Physics Letters, vol. 85, no. 7, pp. 1265-1267, 2004.

[7] E. Luais, M. Boujtita, A. Gohier et al., "Carbon nanowalls as material for electrochemical transducers," Applied Physics Letters, vol. 95, no. 1, Article ID 014104, 2009.

[8] M. Hiramatsu, Y. Nihashi, H. Kondo, and M. Hori, "Nucleation control of carbon nanowalls using inductively coupled plasma-enhanced chemical vapor deposition," Japanese Journal of Applied Physics, vol. 52, no. 1, Article ID 01AK05, 2013.

[9] T.-C. Hung, C.-F. Chen, and W.-T. Whang, "Deposition of carbon nanowall flowers on two-dimensional sheet for electrochemical capacitor application," Electrochemical and Solid-State Letters, vol. 12, no. 6, pp. K41-K44, 2009.

[10] Y. Shiratori, H. Hiraoka, and M. Yamamoto, "Vertically aligned carbon nanotubes produced by radio-frequency plasmaenhanced chemical vapor deposition at low temperature and their growth mechanism," Materials Chemistry and Physics, vol. 87, no. 1, pp. 31-38, 2004.

[11] J. Datta, H. S. Biswas, P. Rao et al., "Study of depth profile of hydrogen in hydrogenated diamond like carbon thin film using ion beam analysis techniques," Nuclear Instruments and Methods in Physics Research, Section B: Beam Interactions with Materials and Atoms, vol. 328, pp. 27-32, 2014. 
[12] C. Surdu-Bob, R. Vladoiu, M. Badulescu, and G. Musa, "Control over the $\mathrm{sp} 2 / \mathrm{sp} 3$ ratio by tuning plasma parameters of the Thermoionic Vacuum Arc," Diamond and Related Materials, vol. 17, no. 7-10, pp. 1625-1628, 2008.

[13] S. Vizireanu, L. Nistor, M. Haupt, V. Katzenmaier, C. Oehr, and G. Dinescu, "Carbon nanowalls growth by radiofrequency plasma-beam-enhanced chemical vapor deposition," Plasma Processes and Polymers, vol. 5, no. 3, pp. 263-268, 2008.

[14] S. Vizireanu, M. D. Ionita, G. Dinescu, I. Enculescu, M. Baibarac, and I. Baltog, "Post-synthesis carbon nanowalls transformation under hydrogen, oxygen, nitrogen, tetrafluoroethane and sulfur hexafluoride plasma treatments," Plasma Processes and Polymers, vol. 9, no. 4, pp. 363-370, 2012.

[15] S. Vizireanu, B. Mitu, C. R. Luculescu, L. C. Nistor, and G. Dinescu, "PECVD synthesis of 2D nanostructured carbon material," Surface and Coatings Technology, vol. 211, pp. 2-8, 2012.

[16] I. Burducea, M. Straticiuc, D. G. Ghiţa et al., "A new ion beam facility based on a 3 MV Tandetron ${ }^{\mathrm{TM}}$ at IFIN-HH, Romania," Nuclear Instruments and Methods in Physics Research, Section B: Beam Interactions with Materials and Atoms, vol. 359, pp. 12-19, 2015.

[17] M. Mayer, "SIMNRA, a simulation program for the analysis of NRA, RBS and ERDA," Proceedings of the 15th International Conference on the Application of Accelerators in Research and Industry, vol. 475, no. 1, pp. 541-544, 1999.

[18] Y. Wang and M. Nastasi, Handbook of Modern Ion Beam Materials Analysis, Materials Research Society, Pennsylvania, $\mathrm{Pa}, \mathrm{USA}, 2010$.

[19] V. A. Krivchenko, S. A. Evlashin, K. V. Mironovich et al., "Carbon nanowalls: The next step for physical manifestation of the black body coating," Scientific Reports, vol. 3, article no. 3328, 2013.

[20] S. Ghosh, K. Ganesan, S. R. Polaki et al., "Evolution and defect analysis of vertical graphene nanosheets," Journal of Raman Spectroscopy, vol. 45, no. 8, pp. 642-649, 2014.

[21] I. Gouzman, O. Girshevitz, V. Richter, E. Shawat Avraham, C. N. Sukenik, and G. D. Nessim, "High Rate of Hydrogen Incorporation in Vertically Aligned Carbon Nanotubes during Initial Stages of Growth Quantified by Elastic Recoil Detection," Journal of Physical Chemistry C, vol. 119, no. 47, pp. 26726-26733, 2015.

[22] F. U. Naab, O. W. Holland, J. L. Duggan, and F. D. McDaniel, "Ion beam analyses of carbon nanotubes," Journal of Physical Chemistry B, vol. 109, no. 4, pp. 1415-1419, 2005.

[23] A. Gohier, S. Point, M. A. Djouadi et al., "ERDA and structural characterization of oriented multiwalled carbon nanotubes," Journal of Physical Chemistry C, vol. 111, no. 28, pp. 10353-10358, 2007.

[24] H. G. Jain, H. Karacuban, D. Krix, H.-W. Becker, H. Nienhaus, and V. Buck, "Carbon nanowalls deposited by inductively coupled plasma enhanced chemical vapor deposition using aluminum acetylacetonate as precursor," Carbon, vol. 49, no. 15, pp. 4987-4995, 2011.

[25] I. Burducea, L. S. Craciun, C. Ionescu, M. Straticiuc, A. T. Serban, and P. M. Racolta, "Nanomaterials characterization using nuclear methods at IFIN-HH," Sensors \& Transducers Journal, vol. 12, pp. 33-45, 2011.

[26] R. A. Quinlan, M. Cai, R. A. Outlaw, S. M. Butler, J. R. Miller, and A. N. Mansour, "Investigation of defects generated in vertically oriented graphene," Carbon, vol. 64, pp. 92-100, 2013.
[27] S. Vizireanu, M. D. Ionita, R. E. Ionita et al., "Aging phenomena and wettability control of plasma deposited carbon nanowall layers," Plasma Processes and Polymers, p. 1700023, 2017.

[28] E. Fuente, J. A. Menéndez, M. A. Díez, D. Suárez, and M. A. Montes-Morán, "Infrared spectroscopy of carbon materials: a quantum chemical study of model compounds," Journal of Physical Chemistry B, vol. 107, no. 26, pp. 6350-6359, 2003.

[29] G.-H. Moon, Y. Park, W. Kim, and W. Choi, "Photochemical loading of metal nanoparticles on reduced graphene oxide sheets using phosphotungstate," Carbon, vol. 49, no. 11, pp. 3454-3462, 2011.

[30] S.-C. Her and C.-Y. Lai, "Dynamic behavior of nanocomposites reinforced with multi-walled carbon nanotubes (MWCNTs)," Materials, vol. 6, no. 6, pp. 2274-2284, 2013.

[31] S. Kurita, A. Yoshimura, H. Kawamoto et al., "Raman spectra of carbon nanowalls grown by plasma-enhanced chemical vapor deposition," Journal of Applied Physics, vol. 97, no. 10, Article ID 104320, 2005.

[32] P. Serp and B. Machado, "Nanostructured Carbon Materials for Catalysis," Royal Society of Chemistry, vol. 40, no. 10, pp. 880881, 2015.

[33] A. Achour, S. Vizireanu, G. Dinescu, L. Le Brizoual, M.-A. Djouadi, and M. Boujtita, "Electrochemical anodic oxidation of nitrogen doped carbon nanowall films: X-ray photoelectron and Micro-Raman spectroscopy study,' Applied Surface Science, vol. 273, pp. 49-57, 2013.

[34] V. Datsyuk, M. Kalyva, K. Papagelis et al., "Chemical oxidation of multiwalled carbon nanotubes," Carbon, vol. 46, no. 6, pp. 833-840, 2008.

[35] T. Uchida, A. Baliyan, T. Fukuda, Y. Nakajima, and Y. Yoshida, "Charged particle-induced synthesis of carbon nanowalls and characterization," RSC Advances, vol. 4, no. 68, pp. 36071-36078, 2014.

[36] Y. H. Wu, T. Yu, and Z. X. Shen, "Two-dimensional carbon nanostructures: Fundamental properties, synthesis, characterization, and potential applications," Journal of Applied Physics, vol. 108, no. 7, Article ID 071301, 2010.

[37] Z. H. Ni, H. M. Fan, Y. P. Feng, Z. X. Shen, B. J. Yang, and Y. H. $\mathrm{Wu}$, "Raman spectroscopic investigation of carbon nanowalls," Journal of Chemical Physics, vol. 124, no. 20, Article ID 204703, 2006.

[38] A. C. Ferrari, "Raman spectroscopy of graphene and graphite: disorder, electron-phonon coupling, doping and nonadiabatic effects," Solid State Communications, vol. 143, no. 1-2, pp. 47-57, 2007.

[39] G. Srinivas, Y. Zhu, R. Piner, N. Skipper, M. Ellerby, and R. Ruoff, "Synthesis of graphene-like nanosheets and their hydrogen adsorption capacity," Carbon, vol. 48, no. 3, pp. 630-635, 2010. 

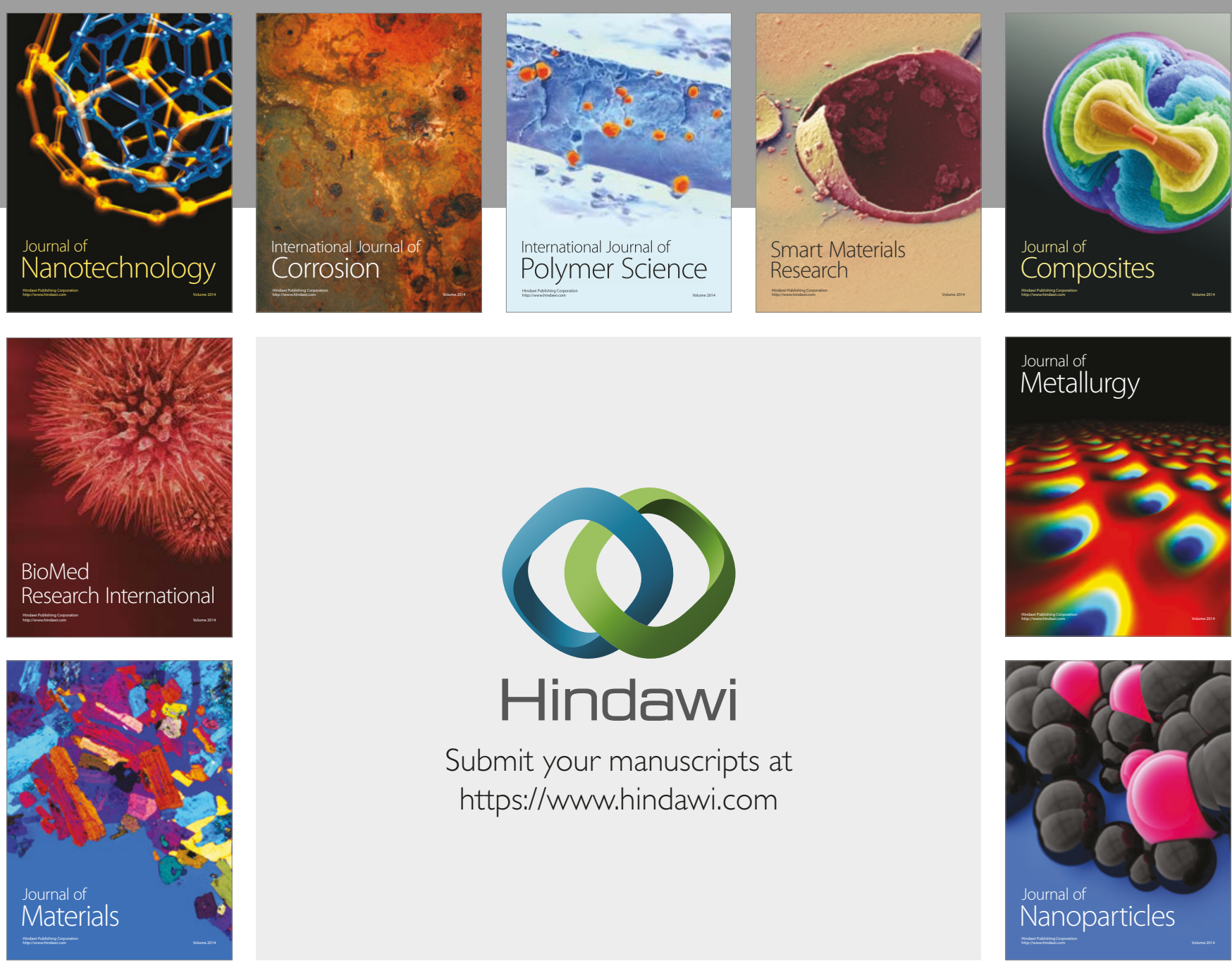

\section{Hindawi}

Submit your manuscripts at

https://www.hindawi.com
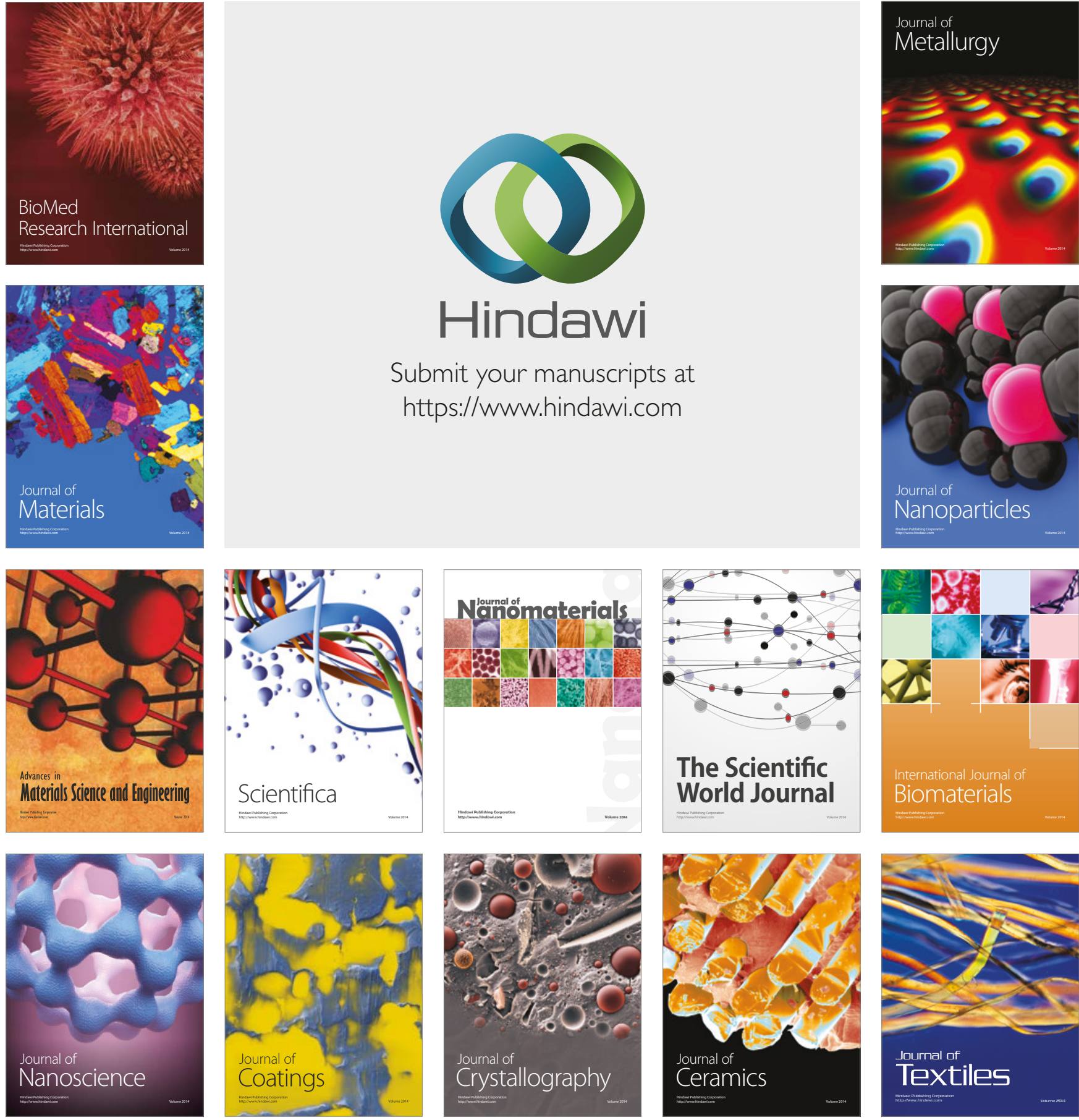

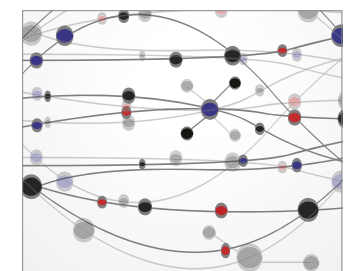

The Scientific World Journal
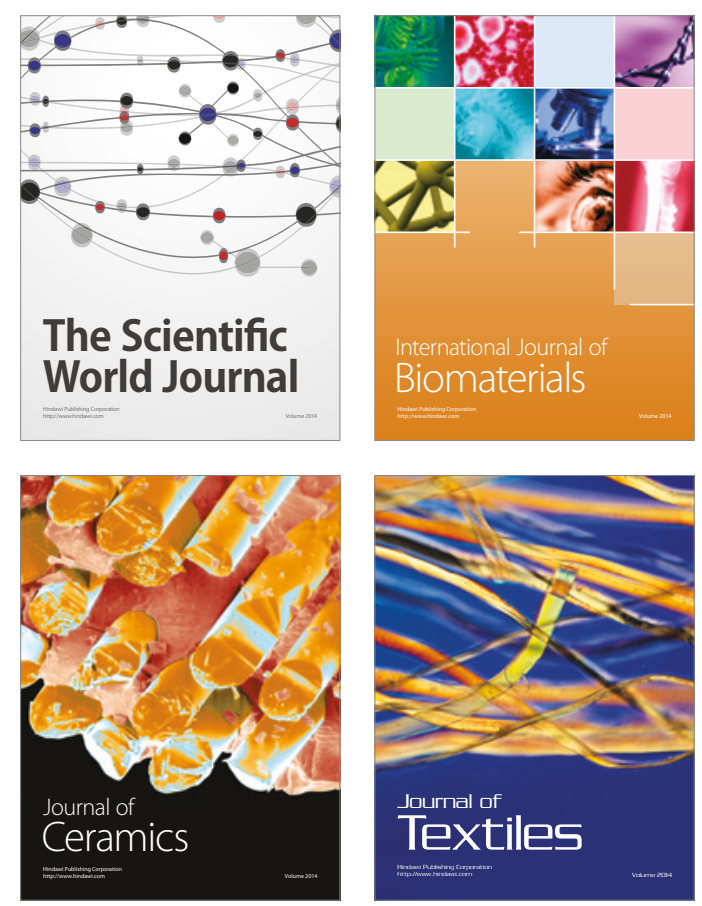